\title{
Aquatic Coleoptera assemblages in protected wetlands of North-western Spain
}

\author{
Amaia PÉREZ-BILBAO, ${ }^{*}$ Cesar João BENETTI, Josefina GARRIDO \\ Department of Ecology and Animal Biology, Faculty of Biology, University of Vigo, 36310 Vigo, Spain \\ *Corresponding author: amaiapb@uvigo.es
}

\begin{abstract}
Wetlands are diverse and productive ecosystems endangered by human pressure, which degradation implies a biodiversity loss worldwide. Among the biological assemblages of these habitats, aquatic Coleoptera is one of the most diverse and useful groups when assessing the ecological conditions of the ecosystems they inhabit. The aims of the present study were to analyze the diversity and composition of aquatic Coleoptera assemblages in 24 wetlands protected by the Natura 2000 network of North-western Spain and the influence of environmental variables on the distribution of species, in order to detect differences between the different types of standing water habitats. A total of 11,136 individuals of 105 species belonging to 12 families of aquatic Coleoptera (Gyrinidae, Haliplidae, Noteridae, Paelobiidae, Dytiscidae, Helophoridae, Hydrochidae, Hydrophilidae, Hydraenidae, Scirtidae, Elmidae and Dryopidae) were collected. In general, wetlands presented high richness and diversity values, Dytiscidae and Hydrophilidae having the highest species richness. Most of recorded species have a wide biogeographical distribution and only 12 endemic ones were captured. Cluster and Non-Metric Multi-Dimensional Scaling (NMDS) analyses showed the clustering of the studied ponds and lagoons in four groups based on biological data. In general, the wetlands of each group seem to have distinct aquatic Coleoptera faunas, as showed by the most representative species. A combination of altitude, SST and hydroperiod was the best explaining factor of the distribution of the species throughout the study area. This study shows the high biodiversity of standing water habitats in North-western Spain and the usefulness of water beetles in establishing habitat typologies.
\end{abstract}

Key words: wetlands, aquatic Coleoptera, diversity, assemblage composition, environmental factors, Natura 2000, NW Spain.

Received: March 2013. Accepted: September 2013.

\section{INTRODUCTION}

Wetlands are sites of high biodiversity and productivity (Mitsch and Gosselink, 2000), but these ecosystems have undergone a serious decline worldwide due to human activity (Shine and Klemm, 1999; Stenert and Maltchik, 2007). In Spain, it was estimated that in the late twentieth century more than $60 \%$ of wetlands had disappeared (Casado and Montes, 1995). The need to protect these ecosystems and many others led to the creation of the Natura 2000 network in Europe. This network is the most important conservation and management tool in the European Union. It was established under the Habitats Directive (92/43/EEC) and the Birds Directive (79/409/EEC), and its main objective is to ensure the long-term conservation of the most important European species and habitats in a sustainable way with human activities. It is formed by Special Areas of Conservation (SACs), which are protected areas established with the purpose of conservation of habitat types and/or species included in the Habitats and Birds directives. In order to apply appropriate protection and management measures it is necessary to determine species diversity and the environmental factors influencing it (Hinden et al., 2005). Studies focusing on wetlands (including faunal and environmental data) have increased worldwide during recent years, e.g. Angélibert et al. (2004), Garrido and Munilla (2008) or Oertli et al. (2008) in Europe; Batzer et al. (2004) or Studinski and Grubbs (2007) in North America; and Stenert and Maltchik (2007) or Burroni et al. (2011) in South America.

Protection measures for wetlands are usually based on a few biological groups (birds, mammals, amphibians, etc.), but for the correct assessment of the ecological status of these ecosystems it is necessary to include studies of the invertebrate fauna. Water beetles are one of the most important invertebrate groups in freshwater ecosystems. Beetles represent the world's most speciose animal order, with more than 13,000 water beetle species described so far, due to their great variety of morphological and ecological adaptations (Jäch and Balke, 2008). The Iberian Peninsula is a recognized hotspot for biodiversity and more than 500 species of aquatic beetles have been reported in this region. Several authors have suggested the use of aquatic Coleoptera as indicators for monitoring population trends in other groups of aquatic macroinvertebrates and for identifying high biodiversity areas at a regional scale (Foster et al., 1990; Sánchez-Fernández et al., 2004, 2006; Guareschi et al., 2012).

This paper studies the diversity and composition of aquatic Coleoptera assemblages in 24 wetlands protected 
by the Natura 2000 network of North-western Spain and the influence of environmental variables on the distribution of species, in order to detect differences between the different types of standing water habitats. Biological and environmental data were used to reach three main objectives: i) to analyze the composition of aquatic Coleoptera assemblages in the protected wetlands; ii) to assess differences between wetlands regarding biological data; and iii) to determine the main environmental factors influencing species distribution.

\section{METHODS}

\section{Study area}

The Autonomous Community of Galicia is located in North-western Spain. According to the Köppen-Geiger Climate Classification, the climate is warm temperate, with dry summers and mild temperature (Kottek et al., 2006). This territory belongs to the Atlantic and the Mediterranean biogeographical regions, with a total area of $29,574 \mathrm{~km}^{2}$ and more than $1200 \mathrm{~km}$ of coastline distributed between the Atlantic Ocean and the Cantabrian Sea. Altitude ranges from the coast to more than $2000 \mathrm{~m}$ in the mountains. Due to its geographical location and orography Galicia has a great variety of aquatic ecosystems.

According to the Spanish Ministry of Agriculture, Alimentation and Environment (MAGRAMA, 2013), in relation to its total area this region has the lowest percentage of its territory protected by the Natura 2000 network $(11.66 \%)$, with a total of $59 \mathrm{SACs}$, of which 26 have standing water habitats.

\section{Sampling}

Twenty four stagnant water bodies belonging to 17 SAC were selected (Fig. 1). The survey was carried out in spring (April-May) of 2007 and 2008. Water bodies were sampled twice, once each year. The landscape around the sampling points varied from crops to bushes, grassland, peat bogs or dunes, and presented different land uses such as industries, cattle rearing, wind farms or small villages.

Aquatic Coleoptera were sampled using an entomological net $(500 \mu \mathrm{m}$ mesh, $30 \mathrm{~cm}$ diameter and $60 \mathrm{~cm}$ deep). Sampling was time-limited. Three minutes total sampling time for each wetland was split equally between the different meso-habitat types of the shore (Biggs et al., 1998). The material was preserved in $99 \%$ ethanol, and

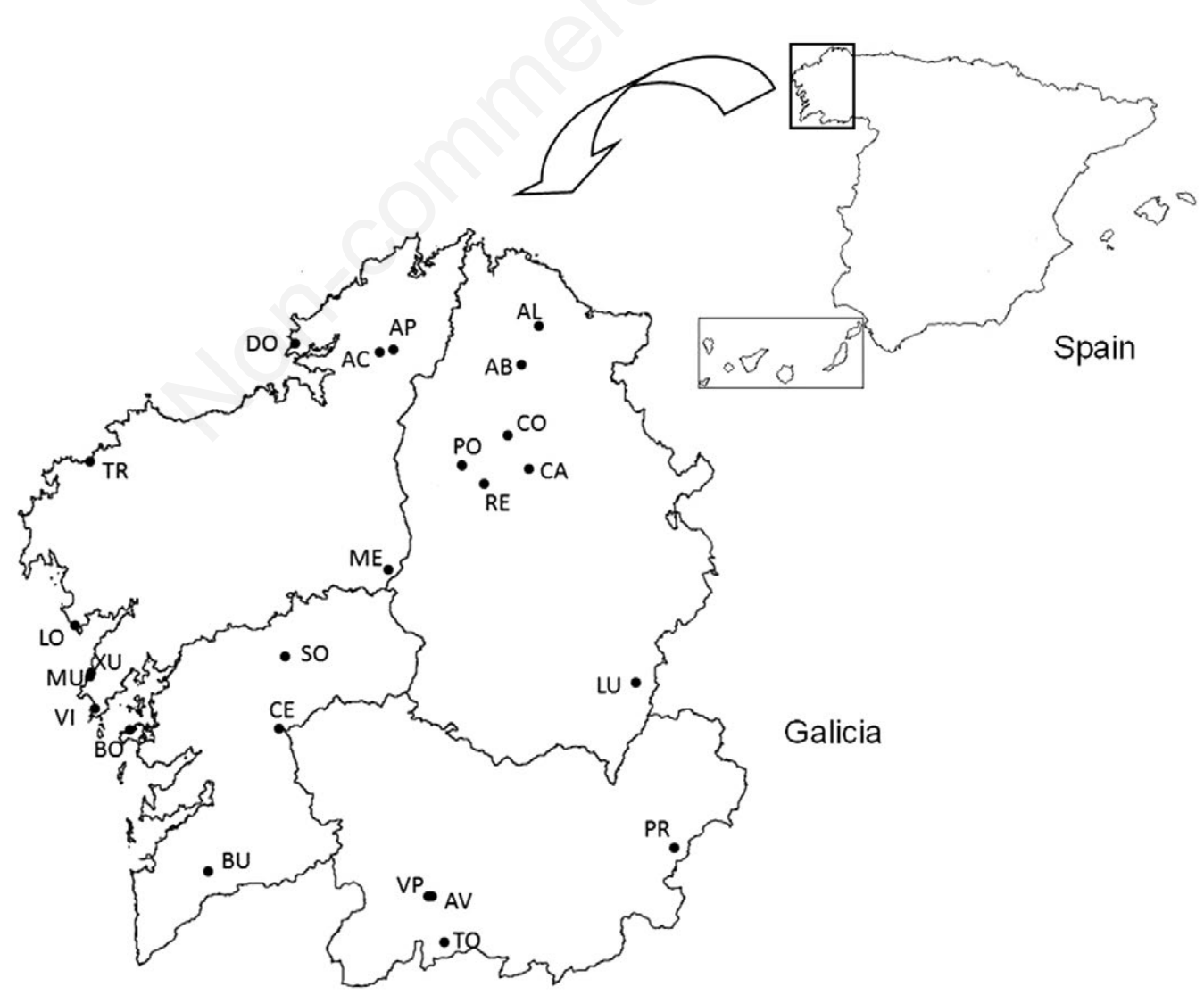

Fig. 1. Map of Galicia (North-western Spain) showing the location of the sampled wetlands. 
sorted and identified to species level at the laboratory. All the specimens are deposited in the collection of the Aquatic Entomology Lab of the University of Vigo.

Several habitat characteristics, such as altitude ( $m$ asl), distance to the sea $(\mathrm{km})$ and percentage of macrophyte cover were considered (Tab. 1). Water permanence (hydroperiod) was included as 1 (permanent) or 2 (temporary), considering water bodies usually dry in summer as temporary. This was visually observed in summer surveys not included in this study. A set of different physical and chemical water variables were measured in situ at each sampling point with field meters (oximeter OXI 330/SET, pH-meter 330/SET WTW and conductivity meter LF 320/SET WTW). These variables were water temperature $\left({ }^{\circ} \mathrm{C}\right), \mathrm{pH}$, conductivity $\left(\mu \mathrm{S} \mathrm{cm}^{-1}\right)$, dissolved oxygen $\left(\mathrm{mg} \mathrm{L}^{-1}\right)$ and total suspended solids (SST) $\left(\mathrm{mg} \mathrm{L}^{-1}\right)$ (Tab. 2). Water samples were also collected in plastic bottles and nitrates $\left(\mathrm{NO}_{3}\right)$, ammonium $\left(\mathrm{NH}_{4}\right)$, total nitrogen ( $\mathrm{N}$ tot) and total phosphorus (P tot) were measured at the Zooplankton Lab of the University of Vigo. Nutrients are expressed in ppm.

\section{Data analysis}

According to the chorological scheme proposed by Ribera et al. (1999) and modified by Fery and Fresneda (2007), the collected species were assigned to biogeographical categories: Trans-Pyrenean (N), Southern (S), Trans-Iberian (T) and Endemic (X).
The parameters used to assess the structure of the assemblages were abundance, species richness and diversity, calculated using the Shannon index $\left(\mathrm{H}^{\prime} \log _{2}\right)$.

For multivariate analysis species with only one individual were removed from the data matrix, considering that a species was present in a site when more than one individual was collected. Biotic data were transformed through square root transformation to reduce the weight of the very abundant species, while environmental variables were normalized. Analyses were carried out on total abundances of species and mean values of the environmental variables for the two years (2007-2008) with PRIMER \& PERMANOVA+ software version 6 (Primer-E Ltd). Similarity relationships among Coleoptera assemblages in all sites were determined by the Bray-Curtis coefficient. Groups of similar sites in terms of Coleoptera composition were identified by Cluster analysis (group average mode) and graphically presented using Non-Metric Multi-Dimensional Scaling (NMDS) mapped in two dimensions.

To investigate the groups consistency the SIMilarity PERcentages-species contributions (SIMPER) analysis was used to obtain differences between all pairs of groups and the contribution of each species for the groups. SIMPER examines the contribution of each species to the average Bray-Curtis dissimilarity between groups of samples and determines the contribution to similarity within a group (Clarke and Warwick, 2001).

To determine whether the groups differed significantly

Tab. 1. Habitat characteristics of each wetland. Percentage of macrophyte cover is presented as mean value of the two years of sampling.

\begin{tabular}{|c|c|c|c|c|c|}
\hline Sampling site & Code & Altitude (m asl) & Distance to the sea $(\mathrm{km})$ & Hydroperiod & Macrophyte cover (\%) \\
\hline Abadín & $\mathrm{AB}$ & 636 & 26 & 2 & 80 \\
\hline A Capela & $\mathrm{AC}$ & 524 & 14 & 2 & 90 \\
\hline Alfoz & $\mathrm{AL}$ & 62 & 13 & 1 & 80 \\
\hline As Pontes & $\mathrm{AP}$ & 434 & 20 & 1 & 50 \\
\hline A Veiga & $\mathrm{AV}$ & 620 & 70 & 2 & 95 \\
\hline Bodeira & $\mathrm{BO}$ & 11 & 0.13 & 1 & 45 \\
\hline Budiño & $\mathrm{BU}$ & 28 & 16 & 1 & 70 \\
\hline Caque & $\mathrm{CA}$ & 425 & 48 & 1 & 30 \\
\hline Cerdedo & $\mathrm{CE}$ & 942 & 25 & 2 & 70 \\
\hline Cospeito & $\mathrm{CO}$ & 407 & 40 & 1 & 60 \\
\hline Doniños & DO & 0 & 0.45 & 1 & 40 \\
\hline Louro & LO & 13 & 0.25 & 1 & 90 \\
\hline Lucenza & LU & 1380 & 105 & 2 & 95 \\
\hline Melide & ME & 452 & 48 & 2 & 85 \\
\hline Muro & MU & 11 & 0.4 & 1 & 20 \\
\hline Pozo do Ollo & $\mathrm{PO}$ & 422 & 45 & 1 & 10 \\
\hline Pradorramisquedo & PR & 1452 & 135 & 1 & 10 \\
\hline Rey & $\mathrm{RE}$ & 418 & 50 & 1 & 85 \\
\hline Sacra de Olives & $\mathrm{SO}$ & 678 & 35 & 2 & 100 \\
\hline Tosende & TO & 868 & 80 & 1 & 90 \\
\hline Traba & TR & 2 & 0.43 & 1 & 15 \\
\hline Vixán & VI & 7 & 0.38 & 1 & 60 \\
\hline Veiga da Pencha & VP & 625 & 70 & 2 & 95 \\
\hline Xuño & $\mathrm{XU}$ & 14 & 0.3 & 1 & 95 \\
\hline
\end{tabular}

Hydroperiod: 1, permanent; 2, temporary. 
in terms of biotic data, we used the Analysis of Similarity (ANOSIM) with a significance level of 0.05 , considering the cluster analysis grouping. This is a simple non-parametric permutation procedure, which is applied to the rank similarity matrix underlying the ordination or classification of samples. The null hypothesis is that there are no differences in community composition of the groups. The procedure computes a test statistic (R), which is close to unity if there is complete segregation between groups and close to zero if there is little or no segregation, and a significance level (t) (Clarke and Warwick, 2001).

A distance-based redundancy analysis (dbRDA) was done to investigate the relation between the assemblages and the environmental variables. This is a method for carrying out constrained ordinations on data using non-Euclidean distance measures (Anderson et al., 2008).

\section{RESULTS}

A total of 11,122 individuals of 105 species belonging to 12 families of aquatic Coleoptera (Gyrinidae, Haliplidae, Noteridae, Paelobiidae, Dytiscidae, Helophoridae, Hydrochidae, Hydrophilidae, Hydraenidae, Scirtidae, Elmidae and Dryopidae) were collected (Tab. 3). The most frequent species in the study area were Helochares (Helochares) punctatus and Noterus laevis, while the most abundant ones were Helophorus (Rhopalhelophorus) flavipes and Ochthebius (Ochthebius) viridis fallaciosus.The most representa- tive biogeographical category was the Trans-Iberian $(67 \%)$, followed by Trans-Pyrenean (19\%), Endemics (12\%) and Southern (2\%) (Fig. 2). Dytiscidae were the best represented family in the Trans-Iberian category (48\%). Among the 12 Iberian endemic species, 5 belonged to the Dytiscidae family, 3 Helophoridae, 3 Hydraenidae and 1 Elmidae. Families with the highest values of species richness were Dytiscidae (47) and Hydrophilidae (19). Richness reached values

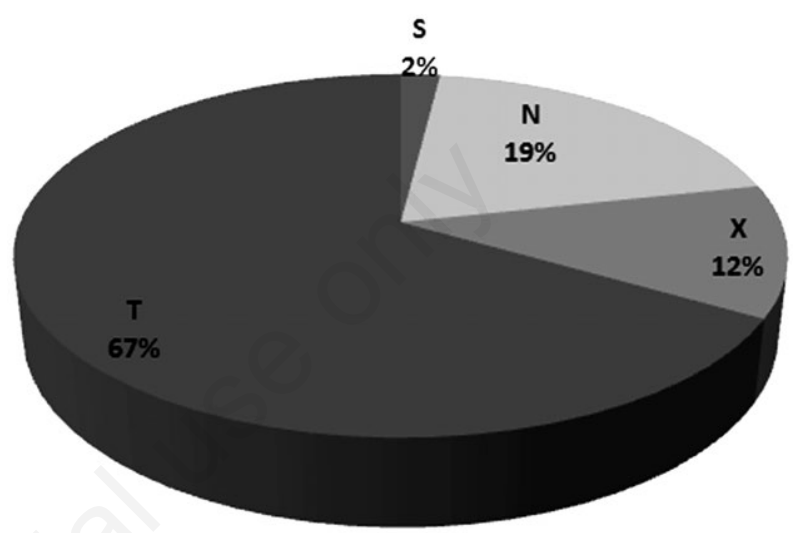

Fig. 2. Percentages of each biogeographical category of the species found in the studied wetlands. T, trans-Iberian; X, endemic; N, trans-Pyrenean; S, southern.

Tab. 2. Mean values of the environmental factors measured in each wetland.

\begin{tabular}{|c|c|c|c|c|c|c|c|c|c|}
\hline Sampling site & $\begin{array}{c}\text { Water } \\
\text { temperature } \\
\left({ }^{\circ} \mathrm{C}\right)\end{array}$ & $\mathrm{pH}$ & $\begin{array}{l}\text { Conductivity } \\
\left(\mu \mathrm{S} \mathrm{cm}^{-1}\right)\end{array}$ & $\begin{array}{l}\text { Dissolved } \\
\text { oxygen } \\
\left(\mathrm{mg} \mathrm{L}^{-1}\right)\end{array}$ & $\begin{array}{c}\mathrm{SST} \\
\left(\mathrm{mg} \mathrm{L}^{-1}\right)\end{array}$ & $\begin{array}{l}\mathrm{NO}_{3} \\
\text { (ppm) }\end{array}$ & $\begin{array}{l}\mathrm{NH}_{4} \\
\text { (ppm) }\end{array}$ & $\begin{array}{l}\text { P tot } \\
(\mathrm{ppm})\end{array}$ & $\begin{array}{l}\mathrm{N} \text { tot } \\
(\mathrm{ppm})\end{array}$ \\
\hline Abadín & 14.7 & 7.47 & 133.9 & 7.9 & 70 & 0.32 & 0.03 & 0.02 & 0.26 \\
\hline A Capela & 15.35 & 5.14 & 45 & 8.18 & 23 & 0.39 & 0.07 & 0.06 & 0.94 \\
\hline Alfoz & 14.6 & 6.39 & 57,8 & 7.55 & 34 & 1.25 & 0 & 0,002 & 0.68 \\
\hline As Pontes & 13.45 & 6.17 & 660 & 8.84 & 345.5 & 0.41 & $<0.01$ & 0.02 & 0.25 \\
\hline A Veiga & 15.8 & 6.08 & 33.7 & 12.15 & 18 & 0.26 & 0.01 & 0.01 & 0.34 \\
\hline Bodeira & 21.1 & 7.13 & 292.5 & 8.4 & 156 & 0.09 & 0.01 & 0.02 & 0.59 \\
\hline Budiño & 15.3 & 6.5 & 266 & 3.61 & 142 & 0.55 & 0.29 & 0.03 & 0.44 \\
\hline Caque & 21.4 & 5.61 & 39.6 & 8.17 & 20 & 0.17 & 0.01 & 0.01 & 0.54 \\
\hline Cerdedo & 11.1 & 5.75 & 21.15 & 12.5 & 11.5 & 2.4 & 0.05 & 0.01 & 1.05 \\
\hline Cospeito & 14.5 & 6.67 & 63.6 & 6.55 & 32.5 & 2.88 & 0.06 & 0.01 & 0.97 \\
\hline Doniños & 18.8 & 7.18 & 229 & 9,05 & 120 & 1.47 & 0.03 & 0.03 & 0.69 \\
\hline Louro & 20.9 & 6.66 & 1050.5 & 5.53 & 1270 & 0.09 & 0.01 & $<0.01$ & 0.37 \\
\hline Lucenza & 13.25 & 6.36 & 13.45 & 10.6 & 7 & 0.38 & $<0.01$ & 0.02 & 0.29 \\
\hline Melide & 15.6 & 7.18 & 92.1 & 10.5 & 49 & 0.22 & $<0.01$ & 0.01 & 0.46 \\
\hline Muro & 24.05 & 7.92 & 440.32 & 11.9 & 508 & 0.99 & 0.01 & 0.02 & 0.76 \\
\hline Pozo do Ollo & 13.55 & 7.14 & 79 & 8.76 & 39.5 & 0.31 & $<0.01$ & 0.02 & 0.31 \\
\hline Pradorramisquedo & 10.5 & 6.44 & 9.35 & 10.85 & 5.5 & 0.34 & 0.01 & 0.01 & 0.15 \\
\hline Rey & 25.3 & 8.07 & 61.15 & 9.27 & 31 & 0.11 & $<0.01$ & 0.02 & 0.76 \\
\hline Sacra de Olives & 17.4 & 7.01 & 14.8 & 9.8 & 7.5 & 0.59 & 0.02 & 0.05 & 0.58 \\
\hline Tosende & 14.05 & 6.15 & 34.5 & 10 & 19 & 0.28 & 0.06 & 0.02 & 0.37 \\
\hline Traba & 17.9 & 7.16 & 213 & 10.15 & 114 & 2.83 & 0 & 0.01 & 0.72 \\
\hline Vixán & 17.6 & 6.67 & 419.2 & 2.64 & 496 & 0.13 & $<0.01$ & 0.03 & 0.96 \\
\hline Veiga da Pencha & 16.65 & 5.96 & 47.75 & 14.4 & 25 & 0.01 & 0 & 0.05 & 0.15 \\
\hline Xuño & 25.6 & 6.76 & 227 & 16.5 & 134 & 0.54 & 0.03 & 0.01 & 0.37 \\
\hline
\end{tabular}


Tab. 3. List of the species collected in the study area.

\begin{tabular}{|c|c|c|c|c|}
\hline Family Species & Category & Family & Species & Category \\
\hline \multicolumn{2}{|l|}{ Gyrinidae } & \multicolumn{3}{|c|}{ Helophoridae } \\
\hline Gyrinus (Gyrinus) caspius Ménétriés, 1832 & $\mathrm{~T}$ & & Helophorus (Trichohelophorus) alternans Gené, 1836 & $\mathrm{~T}$ \\
\hline Gyrinus (Gyrinus) substriatus Stephens, 1828 & $\mathrm{~T}$ & & Helophorus (Helophorus) maritimus Rey, 1885 & $\mathrm{~N}$ \\
\hline Gyrinus (Gyrinus) urinator Illiger, 1807 & $\mathrm{~T}$ & & Helophorus (Atracthelophorus) bameuli Angus, 1987 & $\mathrm{X}$ \\
\hline \multicolumn{2}{|l|}{ Haliplidae } & & Helophorus (Rhopalhelophorus) flavipes Fabricius, & $\mathrm{N}$ \\
\hline Haliplus (Haliplus) heydeni Wehncke, 1875 & $\mathrm{~N}$ & & Helophorus (Rhopalhelophorus) jocoteroi Angus & $\mathrm{N}$ \\
\hline Haliplus (Liaphlus) guttatus Aubé, 1836 & $\mathrm{~T}$ & & \& Díaz Pazos, 1991 & $\mathrm{X}$ \\
\hline Haliplus (Neohaliplus) lineatocollis (Marsham, 1802) & $\mathrm{T}$ & & Helophorus (Rhopalhelophorus) lapponicus Thomson, & \\
\hline Peltodytes caesus (Duftschmid, 1805) & $\mathrm{T}$ & & 1853 & $\mathrm{~N}$ \\
\hline Peltodytes rotundatus (Aubé, 1836) & $\mathrm{T}$ & & Helophorus (Rhopalhelophorus) minutus Fabricius, & $N$ \\
\hline \multicolumn{2}{|l|}{ Noteridae } & & 1775 & $\mathrm{~N}$ \\
\hline Noterus laevis Sturm, 1834 & $\mathrm{~T}$ & & Helophorus (Rhopalhelophorus) seidlitzii Kuwert, 1885 & $\mathrm{X}$ \\
\hline \multicolumn{2}{|l|}{ Paelobiidae } & \multicolumn{3}{|c|}{ Hydrochidae } \\
\hline Hygrobia hermanni (Fabricius, 1775) & $\mathrm{T}$ & & & $\mathrm{T}$ \\
\hline \multirow{2}{*}{\multicolumn{2}{|c|}{ Dytiscidae }} & \multirow{2}{*}{\multicolumn{2}{|c|}{$\begin{array}{l}\text { Hydrochus flavipennis Küster, } 1852 \\
\text { Hydrochus nitidicollis Mulsant, } 1844\end{array}$}} & $\mathrm{~T}$ \\
\hline & $\mathrm{N}$ & & & $\mathrm{T}$ \\
\hline Agabus (Gaurodytes) brunneus (Fabricius, 1798) & $\mathrm{T}$ & \multicolumn{3}{|c|}{ Hydrophilidae } \\
\hline Agabus (Gaurodytes) didymus (Olivier, 1795) & $\mathrm{T}$ & & Berosus (Berosus) affinis Brullé, 1835 & $\mathrm{~T}$ \\
\hline Agabus (Gaurodytes) biguttatus (Olivier, 1795) & $\mathrm{T}$ & & Berosus (Berosus) hispanicus Küster, 1847 & $\mathrm{~T}$ \\
\hline Agabus (Gaurodytes) conspersus (Marsham, 1802) & $\mathrm{T}$ & & Berosus (Berosus) signaticollis (Charpentier, 1825) & $\mathrm{T}$ \\
\hline Agabus (Gaurodytes) nebulosus (Forster, 1771) & $\mathrm{T}$ & & Paracymus scutellaris (Rosenhauer, 1856) & $\mathrm{T}$ \\
\hline Agabus (Gaurodytes) paludosus (Fabricius, 1801) & $\mathrm{N}$ & & Anacaena bipustulata (Marsham, 1802) & $\mathrm{T}$ \\
\hline Agabus (Gaurodytes) bipustulatus (Linnaeus, 1767) & $\mathrm{T}$ & & Anacaena globulus (Paykull, 1798) & $\mathrm{T}$ \\
\hline Ilybius dettneri (Fery, 1986) & $\mathrm{X}$ & & Anacaena lutescens (Stephens, 1829) & $\mathrm{T}$ \\
\hline Ilybius montanus (Stephens, 1828) & $\mathrm{T}$ & \multicolumn{3}{|c|}{ Laccobius (Dimorpholaccobius) atratus Rottenberg, } \\
\hline Ilybius meridionalis Aubé, 1837 & $\mathrm{~T}$ & & 1874 & $\mathrm{~N}$ \\
\hline Colymbetes fuscus (Linnaeus, 1758) & $\mathrm{T}$ & \multicolumn{3}{|c|}{ Laccobius (Dimorpholaccobius) sinuatus Motschulsky, } \\
\hline Rhantus (Rhantus) hispanicus Sharp, 1882 & $\mathrm{~T}$ & & 1849 & $\mathrm{~T}$ \\
\hline Rhantus (Rhantus) suturalis (McLeay, 1825) & $\mathrm{T}$ & & Helochares (Helochares) punctatus Sharp, 1869 & $\mathrm{~N}$ \\
\hline Liopterus haemorrhoidalis (Fabricius, 1787) & $\mathrm{T}$ & & Enochrus (Methydrus) nigritus (Sharp, 1872) & $\mathrm{T}$ \\
\hline Acilius (Acilius) sulcatus (Linnaeus, 1758) & $\mathrm{T}$ & & Enochrus (Lumetus) fuscipennis (Thomson, 1884) & $\mathrm{T}$ \\
\hline \multicolumn{2}{|l|}{ Cybister (Scaphinectes) lateralimarginalis } & & Cymbiodyta marginella (Fabricius, 1792) & $\mathrm{N}$ \\
\hline (De Geer, 1774) & $\mathrm{T}$ & & Hydrobius convexus Brullé, 1835 & $\mathrm{~T}$ \\
\hline Dytiscus marginalis Linnaeus, 1758 & $\mathrm{~N}$ & & Hydrobius fuscipes (Linnaeus, 1758) & $\mathrm{T}$ \\
\hline Dytiscus semisulcatus O.F. Müller, 1776 & $\mathrm{~T}$ & & Limnoxenus niger (Gmelin, 1790) & $\mathrm{N}$ \\
\hline Bidessus minutissimus (Germar, 1824) & $\mathrm{T}$ & & Hydrophilus (Hydrophilus) pistaceus Laporte, 1840 & $\mathrm{~T}$ \\
\hline Bidessus goudoti (Laporte, 1835) & $\mathrm{T}$ & & Coelostoma (Coelostoma) orbiculare (Fabricius, 1775) & $\mathrm{N}$ \\
\hline Hydroglyphus geminus (Fabricius, 1792) & $\mathrm{T}$ & & Cercyon (Cercyon) haemorrhoidalis (Fabricius, 1775) & $\mathrm{T}$ \\
\hline Yola bicarinata (Latreille, 1804) & $\mathrm{T}$ & \multirow{2}{*}{\multicolumn{3}{|c|}{ Hydraenidae }} \\
\hline Graptodytes bilineatus (Sturm, 1835) & $\mathrm{N}$ & & & \\
\hline Graptodytes flavipes (Olivier, 1795) & $\mathrm{T}$ & & Hydraena testacea Curtis, 1830 & $\mathrm{~T}$ \\
\hline Graptodytes fractus (Sharp, 1882) & $\mathrm{T}$ & & Hydraena affusa D’Orchymont, 1936 & $\mathrm{X}$ \\
\hline Graptodytes ignotus (Mulsant \& Rey, 1861) & $\mathrm{T}$ & & Hydraena bisulcata Rey, 1884 & $\mathrm{~S}$ \\
\hline Graptodytes varius (Aubé, 1838) & $\mathrm{T}$ & & Hydraena brachymera D'Orchymont, 1936 & $\mathrm{X}$ \\
\hline Hydroporus vespertinus Fery \& Hendrich, 1988 & $\mathrm{X}$ & & Hydraena rugosa Mulsant, 1844 & $\mathrm{~N}$ \\
\hline \multirow{2}{*}{\multicolumn{2}{|c|}{$\begin{array}{l}\text { Hydroporus discretus discretus Fairmaire \& Brisout, } \\
1859\end{array}$}} & & Limnebius furcatus Baudi, 1872 & $\mathrm{~T}$ \\
\hline & & & Limnebius lusitanus Balfour-Browne, 1978 & $\mathrm{X}$ \\
\hline Hydroporus planus (Fabricius, 1782) & $\mathrm{T}$ & & Ochthebius (Asiobates) dilatatus Stephens, 1829 & $\mathrm{~T}$ \\
\hline Hydroporus pubescens (Gyllenhal, 1808) & $\mathrm{T}$ & & Ochthebius (Ochthebius) viridis fallaciosus Ganglbauer, & \\
\hline Hydroporus brancoi brancoi Rocchi, 1981 & $\mathrm{X}$ & & 1901 & $\mathrm{~T}$ \\
\hline Hydroporus nigrita (Fabricius, 1792) & $\mathrm{N}$ & \multirow{2}{*}{\multicolumn{3}{|c|}{ Scirtidae }} \\
\hline Hydroporus vagepictus Fairmaire \& Laboulbène, 1855 & $\mathrm{X}$ & & & \\
\hline Hydroporus gyllenhalii Schiødte, 1841 & $\mathrm{~N}$ & & Helodes sp. & \\
\hline Metaporus meridionalis (Aubé, 1838) & $\mathrm{T}$ & & Cyphon sp. & \\
\hline Stictonectes epipleuricus (Seidlitz, 1887) & $\mathrm{X}$ & & Hydrocyphon sp. & \\
\hline Stictonectes lepidus (Olivier, 1795) & $\mathrm{T}$ & Elmidae & & \\
\hline Stictotarsus duodecimpustulatus (Fabricius, 1792) & $\mathrm{N}$ & & Dupophilus brevis Mulsant \& Rey, 1872 & $\mathrm{~N}$ \\
\hline Boreonectes ibericus Dutton \& Angus, 2007 & $\mathrm{~N}$ & & Oulimnius bertrandi Berthélemy, 1964 & $\mathrm{X}$ \\
\hline Hydrovatus clypealis Sharp, 1876 & $\begin{array}{l}\mathrm{T} \\
\mathrm{S}\end{array}$ & & Oulimnius rivularis (Rosenhauer, 1856) & $\mathrm{T}$ \\
\hline $\begin{array}{l}\text { Hygrotus (Coelambus) lagari (Fery, 1992) } \\
\text { Hygrotus (Hygrotus) inaequalis (Fabricius, 1777) }\end{array}$ & $\begin{array}{l}\mathrm{S} \\
\mathrm{T}\end{array}$ & Dryopida & & \\
\hline Hyphydrus aubei Ganglbauer, 1891 & $\mathrm{~T}$ & & Dryops algiricus (Lucas, 1846) & $\mathrm{T}$ \\
\hline Laccophilus hyalinus (De Geer, 1774) & $\mathrm{T}$ & & Dryops luridus (Erichson, 1847) & $\mathrm{T}$ \\
\hline Laccophilus minutus (Linnaeus, 1758) & $\mathrm{T}$ & & Dryops striatellus (Fairmaire \& Brisout, 1859) & $\mathrm{T}$ \\
\hline
\end{tabular}

$N$, trans-Pyrenean; $S$, southern; $T$, trans-Iberian; $X$, endemic. 
higher than 30 species in some of the sampling points, for example A Veiga da Pencha (39), Tosende (34), Melide (33), Xuño (32) and A Veiga (31), while in Budiño only 3 species were collected. Different ponds presented high diversity values, for example A Veiga (4.26), Tosende (4.13) or A Veiga da Pencha (3.92). The lowest diversity values corresponded to Cerdedo (1.07) and Budiño (1.18) (Tab. 4).

Four groups were found in the study area based on the
Cluster and NMDS analyses (Fig. 3). The Budiño, Alfoz and Pradorramisquedo lagoons appeared isolated in the clustering of the sites and were not included in any of the groups. The contribution of the species for each group according to the SIMPER analysis is given in Tab. 5. Similarities within each group ranged from $24.43 \%$ to $46.83 \%$. The mean dissimilarities between groups ranged from $73.94 \%$ (groups 2 and 3) to $90.03 \%$ (groups 1 and 4).

\section{a)}

\section{Group average}

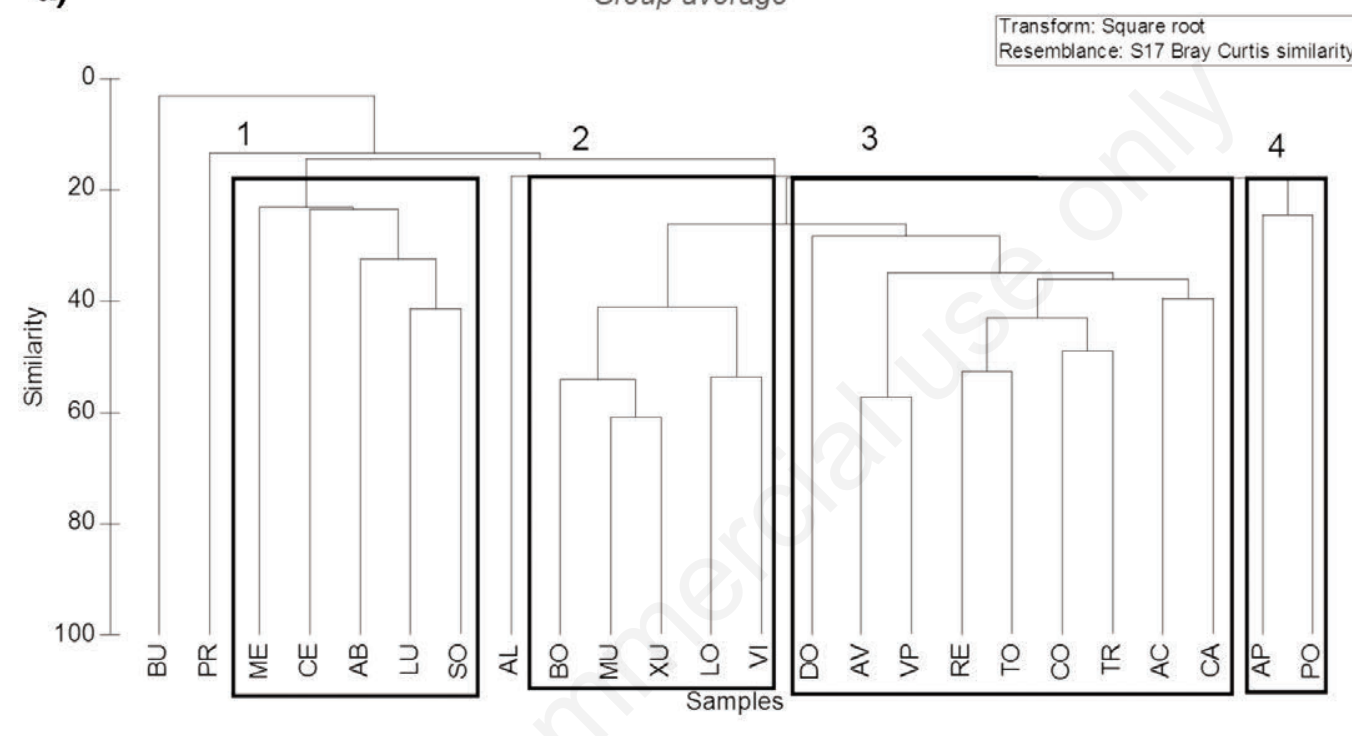

b)

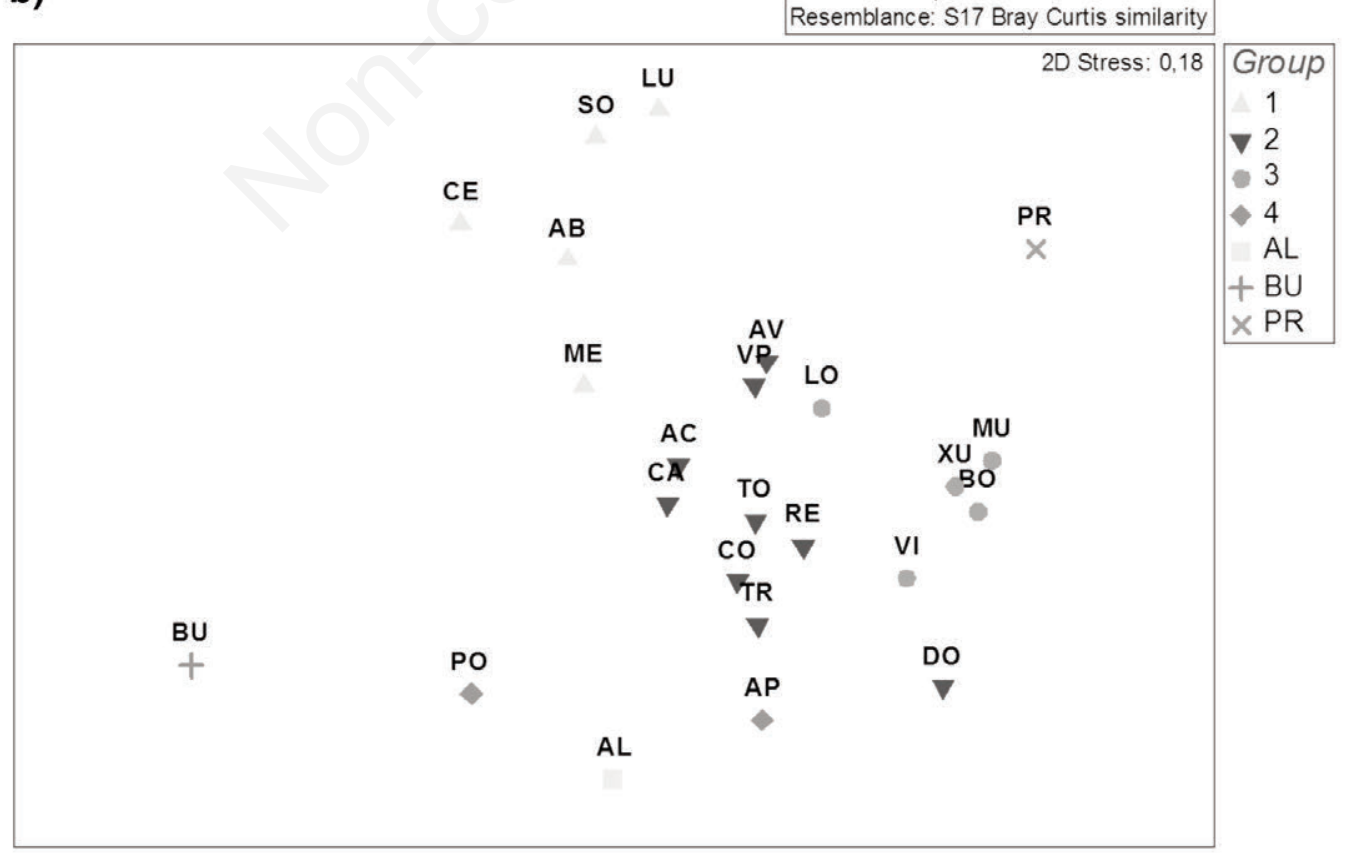

Fig. 3. a) Cluster and b) ordination by Non-Metric Multi-Dimensional Scaling of the studied wetlands. 
These groups were significantly different to each other (ANOSIM, Global test: $\mathrm{R}=0.83, \mathrm{P}=0.001$, 999 permutations) and can be characterized as follows:

Group 1 (within-group similarity: 26.91\%). Formed by medium-high altitude temporary ponds (mean $=817.6$ m) with low conductivity values (mean $=55 \mu \mathrm{S} \mathrm{cm}^{-1}$ ) and high percentage of macrophyte cover (mean $=86 \%$ ). Mainly associated with Helophorus flavipes, H. alternans, Dryops striatellus, Enochrus fuscipennis, $\mathrm{Hy}$ droporus vespertinus, Berosus signaticollis and Agabus bipustulatus.

Group 2 (within-group similarity: 36.17\%). Formed by medium altitude permanent and temporary ponds and lagoons (mean $=432.1 \mathrm{~m}$ ) with medium conductivity values (mean $=85.25 \mu \mathrm{S} \mathrm{cm}^{-1}$ ) and medium percentage of macrophyte cover (mean $=66.6 \%$ ). Mainly associated with Noterus laevis, Helochares punctatus, $H y$ drovatus clypealis, Hygrotus inaequalis, Helophorus alternans and Hydrocyphon sp.

Group 3 (within-group similarity: 46.83\%). Formed by low altitude permanent lagoons (mean=11.2 $\mathrm{m}$ ), near the sea (mean=300 m) with high conductivity values (mean=485.9 $\mu \mathrm{S} \mathrm{cm}^{-1}$ ) and medium percentage of macrophyte cover (mean $=62 \%$ ). Mainly associated with Ochthebius viridis fallaciosus, Limnoxenus niger, Noterus laevis, Hygrotus inaequalis, Enochrus fuscipennis, Limnebius furcatus and Anacaena lutescens.

Group 4 (within-group similarity: 24.43\%). Formed by medium altitude permanent ponds $($ mean $=428 \mathrm{~m})$ with high conductivity values (mean $=369.5 \mu \mathrm{S} \mathrm{cm}^{-1}$ ) and low macrophyte cover (mean $=30 \%$ ). Associated with Laccophilus hyalinus, Dryops luridus, Rhantus suturalis, Agabus bipustulatus and Helochares punctatus. The first two axes of the dbRDA explained the 38.28\% of the cumulative variation in the aquatic Coleoptera assemblages (Fig. 4). The first axis was positively correlated with the altitude $(\mathrm{r}=0.64)$ and the second axis was positively correlated with the SST $(r=0.52)$ and the hydrope- riod $(\mathrm{r}=0.49)$. SST was highly correlated with conductivity $(r=0.94)$ and altitude with distance to the sea $(r=0.88)$ (Draftsman plots; Pearson correlation coefficient). Thus, the environmental variables that best explained the distribution of Coleoptera species throughout the study area were altitude, SST and hydroperiod.

\section{DISCUSSION}

This study contributed to the knowledge and better understanding of diversity of aquatic Coleoptera species in

Tab. 4. Abundance, species richness and diversity values calculated for each wetland.

\begin{tabular}{lccc}
\hline Sampling site & Abundance & Species richness & H'$^{\prime}$ \\
\hline Abadín & 315 & 19 & 2.9 \\
A Capela & 196 & 15 & 2.69 \\
Alfoz & 50 & 11 & 2.61 \\
As Pontes & 227 & 15 & 1.97 \\
A Veiga & 286 & 31 & 4.26 \\
Bodeira & 1286 & 25 & 2.06 \\
Budiño & 12 & 3 & 1.18 \\
Caque & 190 & 22 & 3.03 \\
Cerdedo & 1296 & 20 & 1.07 \\
Cospeito & 308 & 25 & 2.95 \\
Doniños & 195 & 11 & 2.3 \\
Louro & 502 & 29 & 3.22 \\
Lucenza & 114 & 13 & 2.65 \\
Melide & 424 & 33 & 3.27 \\
Muro & 1130 & 23 & 2.63 \\
Pozo do Ollo & 35 & 14 & 3.39 \\
Pradorramisquedo & 385 & 14 & 2.29 \\
Rey & 570 & 26 & 2.53 \\
Sacra de Olives & 331 & 15 & 2.94 \\
Tosende & 338 & 34 & 4.13 \\
Traba & 133 & 19 & 3.29 \\
Vixán & 188 & 23 & 2.78 \\
Veiga da Pencha & 684 & 39 & 3.92 \\
Xuño & 1927 & 32 & 2.93 \\
\hline
\end{tabular}

H', diversity.

Tab. 5. Species that most contributed to the characterization of each group. ${ }^{*}$

\begin{tabular}{|c|c|c|c|}
\hline Group of sites & Number of sites & Most contributive species & Contribution to the group characterization \\
\hline 1 & 5 & $\begin{array}{l}\text { Helophorus flavipes, Helophorus alternans, Dryops } \\
\text { striatellus, Enochrus fuscipennis, Hydroporus vespertinus, } \\
\text { Berosus signaticollis, Agabus bipustulatus }\end{array}$ & $74.24 \%$ (H. flavipes contributed with $27.16 \%$ ) \\
\hline 2 & 9 & $\begin{array}{l}\text { Noterus laevis, Helochares punctatus, Hydrovatus clypealis, } \\
\text { Hygrotus inaequalis, Helophorus alternans, Hydrocyphon sp }\end{array}$ & $55.15 \%(N$. laevis contributed with $19.91 \%)$ \\
\hline 3 & 5 & $\begin{array}{l}\text { Ochthebius viridis fallaciosus, Limnoxenus niger, Noterus laevis, } \\
\text { Hygrotus inaequalis, Enochrus fuscipennis, Limnebius furcatus, } \\
\text { Anacaena lutescens }\end{array}$ & $\begin{array}{l}69.79 \%(O . \text { viridis fallaciosus contributed with } \\
28.65 \%)\end{array}$ \\
\hline 4 & 2 & $\begin{array}{l}\text { Laccophilus hyalinus, Dryops luridus, Rhantus suturalis, } \\
\text { Agabus bipustulatus, Helochares punctatus }\end{array}$ & $100 \%$ (L. hyalinus contributed with $40.46 \%)$ \\
\hline
\end{tabular}

*Performed through the SIMilarity PERcentages-species contributions (SIMPER) analysis. 
north-western Spain. In general, the studied ponds and lagoons showed a great diversity of aquatic Coleoptera. The lowest diversity values were found in Cerdedo and Budiño. In the case of Cerdedo, the high abundance of Helophorus flavipes (1073 individuals) decreased diversity. On the other hand, the low diversity in Budiño was due to the bad conservation state of the lagoon, where only three species were captured. This area is heavily impacted by industrial pollution, as indicated by previous studies (Pérez-Bilbao and Garrido, 2009). In this work, we found high conductivity $\left(266 \mu \mathrm{S} \mathrm{cm}^{-1}\right)$ and ammonium $(0.29 \mathrm{ppm})$ values. These high values for the Budiño lagoon could be pollution indicators in line with other works such as Vivas et al. (2002) or Benetti and Garrido (2010).

Most of the identified species belong to the TransIberian biogeographical category, probably due to the great dispersal ability of the aquatic beetle species typically found in standing water bodies (Arribas et al., 2012). These are more unstable habitats, making species dispersion and migration necessary (Ribera and Vogler, 2000). Although the percentage of endemic species was low $(12 \%)$, some interesting species were identified in this cat- egory. Helophorus jocoteroi (Angus and Díaz-Pazos, 1991; Valladares and Ribera, 1999; Pérez-Bilbao et al., 2011) and Ilybius dettneri (Fresneda et al., 1990; Fery and Nilsson, 1993; Fery and Fresneda, 2007; Pérez-Bilbao et al., 2010) have only been collected in Galicia, north of Portugal and the latter also in Barcelona, while Helophorus bameuli is scarcely cited in the Iberian Peninsula (Valladares and Ribera, 1999; Foster, 2002; Pérez-Bilbao et al., 2011). Another interesting species was Agabus labiatus, which has only been cited in the north of the Iberian Peninsula (González, 1992; González and Novoa, 1995; Valladares and Garrido, 2001; Valladares et al., 2002; Pérez-Bilbao et al., 2010). These species present a narrow geographical and ecological range and are rarely sampled in the Iberian Peninsula.

The variables that best explained the distribution and composition of aquatic beetle assemblages were altitude, SST and hydroperiod. This is explained by the combination of these factors, which formed a gradient from the interior (temporal wetlands at high altitude with low values of conductivity) to the coast (permanent wetlands at low altitude with high values of conductivity) in our study

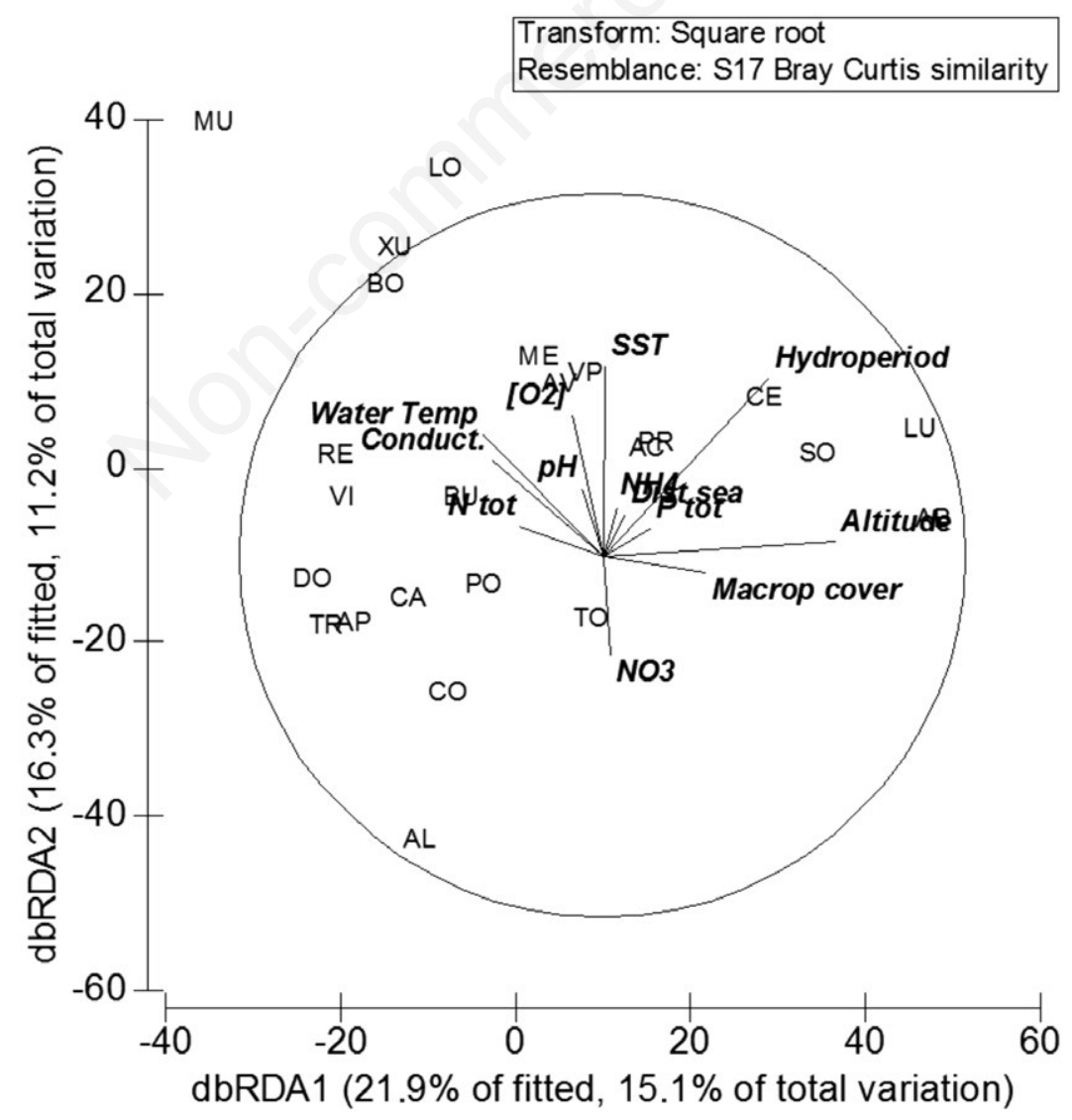

Fig. 4. First two axes of the distance-based redundancy analysis (dbRDA). 
area. This result is in agreement with other studies that found a relationship between these factors and changes in the composition of aquatic beetle assemblages (Valladares et al., 1990, 2002; Eyre et al., 1992; Garrido et al., 1994; Fairchild et al., 2003). However, in the study of Picazo et al. (2012) altitude, conductivity and anthropogenic impact were the most important factors, but hydroperiod did not seem to be a key factor in assemblage composition. This could be due to the number of categories established to measure the factor: three categories (permanent, near-permanent, intermittent) in their study and two (permanent, temporary) in ours, which can create significant differences between categories. According to Boix et al. (2001), hydroperiod is one of the main factors affecting the composition and structure of aquatic assemblages. Organisms that live in temporary waters have to adapt to temporary drought conditions to survive, sometimes being exclusive to these ecosystems (Williams, 2006; Boix et al., 2001; Valladares et al., 2002). Species like Agabus labiatus, Hydroporus nigrita or Helophorus jocoteroi were only collected in temporary habitats, while other species like Hydraena rugosa were very abundant. In this study, species composition in the first group of wetlands identified by the Cluster analysis was clearly influenced by water permanence. It was composed by temporary water bodies located at high altitude with low conductivity values. The species that most contributed to the characterization of this group was Helophorus flavipes, which is widely distributed throughout the Iberian Peninsula. In Galicia, this species seems to be typical of small temporary ponds located at medium-high altitude, although it was also captured in a coastal lagoon, showing a wide ecological range.

The influence of altitude on the distribution of aquatic Coleoptera assemblages has already been demonstrated by several studies (Valladares et al., 1990; Garrido et al., 1994; García-Criado et al., 1999; Picazo et al., 2012). In the present study, two cluster groups seem to be mainly influenced by altitude. The first one is group 3, which is formed by coastal lagoons located at sea level. Due to their transitional character and the interaction with the terrestrial ecosystem, they are high productivity areas (Basset, 2007) with high conductivity values related to salinity (Arnold and Ormerod, 1997; Garrido and Munilla, 2008). In this study, conductivity appeared highly correlated with SST. Conductivity is a good indicator of different types of pollution, like waste waters, mining or fertilizers (de Paz, 1993; García-Criado, 1999; Trigal, 2006), but in the case of the group 3 it can be considered a natural stressor due to the influence of saline water in coastal lagoons. The species that most contributed in this group was Ochthebius viridis fallaciosus, exclusively collected in coastal lagoons and typical of habitats with high values of conductivity or salinity (Valladares et al., 2002; Garrido and Munilla, 2008). Other species typical of coastal lagoons that contributed to the group characterization was Limnebius furcatus, which was only captured in this type of habitat, confirming the results of other studies for this species in Galicia (Díaz-Pazos and Otero, 1993; Garrido and Sáinz-Cantero, 2004; Garrido and Munilla, 2008).

The other group mainly influenced by altitude is group 2, the most heterogeneous one being formed by coastal lagoons, small temporary ponds, big permanent lagoons, etc. Most of the wetlands were located at medium altitude, but it is difficult to say if altitude was the only environmental factor defining the group. Many environmental variables are closely related to altitude and the effects of particular variables are difficult to distinguish (Mendoza and Catalán, 2010). In this study, the species Helophorus alternans, Noterus laevis, Hygrotus inaequalis, Agabus bipustulatus and Enochrus fuscipennis contributed to the characterization of groups with different mean altitude showing a wide ecological range, while other species were captured in mountainous ponds (Helophorus jocoteroi, Boreonectes ibericus, Hydroporus pubescens) or in coastal lagoons (Ochthebius viridis fallaciosus, Limnebius furcatus, Berosus hispanicus, Cymbiodyta marginella, Peltodytes caesus, Hygrotus lagari, Agabus conspersus, Dryops algiricus) showing a narrower ecological range. This suggests a replacement of species, filling different niches in terms of adaptation to habitat characteristics.

The last cluster group (group 4) seems to be related to high conductivity values and low percentage of macrophyte cover. The species that most contributed was Laccophilus hyalinus, which has already been related to high conductivity values by other authors (Martinoy et al., 2006; Pérez-Bilbao and Garrido, 2009).

Although all the wetlands are already protected by the Natura 2000 network, the knowledge of the main environmental factors driving aquatic beetle distribution and the differences regarding biological composition is an interesting result in terms of establishing adequate management measures for each type of aquatic ecosystem. Several studies highlighted the contribution of standing waters to regional freshwater biodiversity (Williams et al., 2003; Picazo et al., 2010) and the usefulness of aquatic Coleoptera as surrogates of biodiversity (Foster et al., 1990; Sánchez-Fernández et al., 2004, 2006; Guareschi et al., 2012). Therefore, management measures in standing water bodies should consider water beetles as indicators, as they are sensitive species to ecological changes and habitat characteristics.

The studied wetlands presented high richness values and some of them constitute the habitat for rare or endemic species in the Iberian Peninsula, giving a high conservation value to these habitats. Bilton et al. (2009) suggested not only the landscape management but also the individual site protection. This could be a very inter- 
esting measure especially for temporary ponds in agricultural areas that can be considered keystone structures for the maintenance of biodiversity (Tews et al., 2004).

Finally, in several of the studied wetlands we found the invasive species Procambarus clarkii (Girard, 1852), the red-clawed crayfish. Protection measures must ensure the absence of invasive species, which can induce the destruction of the habitat for the autochthonous species. Thus, the control of the exotic species must be a priority in conservation policies.

\section{ACKNOWLEDGEMENTS}

This study was supported by the Galician Government with the project Study and assessment of the diversity of aquatic invertebrates in Galician standing waters included in the Natura 2000 network (PGIDIT06RFO31001OR). The authors wish to thank the helpful comments of the anonymous referees.

\section{REFERENCES}

Anderson MJ, Gorley RN, Clarke KR, 2008. PERMANOVA+ for PRIMER: Guide to Software and Statistical Methods. PRIMER-E Ltd., Plymouth Marine Laboratory, UK: 214 pp.

Angélibert S, Marty P, Céréghino R, Giani N, 2004. Seasonal variations in the physical and chemical characteristics of ponds: implications for biodiversity conservation. Aquat. Conserv. 14:439-456.

Angus RB, Díaz Pazos JA, 1991. Helophorus jocoteroi n. sp., from Northwest Spain (Coleoptera, Hydrophilidae). Nouv. Revue Ent. 7:419-422.

Arnold SL, Ormerod SJ, 1997. Aquatic macroinvertebrates and environmental gradients in Phragmites reedswamps: implications for conservation. Aquat. Conserv. 7:153-163.

Arribas P, Velasco J, Abellán P, Sánchez-Fernández D, Andújar C, Calosi P, Millán A, Ribera I, Bilton D, 2012. Dispersal ability rather than ecological tolerance drives differences in range size between lentic and lotic water beetles (Coleoptera: Hydrophilidae). J. Biogeogr. 39:984-994.

Basset A, 2007. Ecosystems and Society: do they really need to be bridged? Aquat. Conserv. 17:551-553.

Batzer DP, Palik BJ, Buech R, 2004. Relationships between environmental characteristics and macroinvertebrate communities in seasonal woodland ponds of Minnesota. J. N. Am. Benthol. Soc. 23:50-68.

Benetti CJ, Garrido J, 2010. The influence of water quality and stream habitat on water beetle assemblages in two rivers in Northwest Spain. Vie Milieu 60:53-63.

Biggs J, Fox G, Nicolet P, Walker D, Whitfield M, Williams P, 1998. A guide to the methods of the national pond survey. Pond Action, Oxford: 22 pp.

Bilton DT, McAbendroth LC, Nicolet P, Bedford A, Rundle SD, Foggo A, Ramsay PM, 2009. Ecology and conservation status of temporary and fluctuating ponds in two areas of southern England. Aquat. Conserv. 19:134-146.

Boix D, Sala J, Moreno-Amichi R, 2001. The faunal composition of Espolla pond (NE Iberian Peninsula): the neglected biodiversity of temporary waters. Wetlands 21:577-592.
Burroni NE, Marinone MC, Freire MG, Schweigmann N, Loetti MV, 2011. Invertebrate communities from different wetland types of Tierra del Fuego. Insect Conserv. Diver. 4:39-45.

Casado S, Montes C, 1995. [Guía de los lagos y humedales de España].[Book in Spanish]. JM Reyero, Madrid: 255 pp.

Clarke KR, Warwick RM, 2001. Change in marine communities: an approach to statistical analysis and interpretation, 2nd ed. Primer-e Ltd, Plymouth Marine Laboratory: 169 pp.

de Paz C, 1993. [Hydradephaga (Coleoptera) de la cuenca del río Landro (NW Península Ibérica). Estudio faunístico y ecológico]. [PhD Thesis in Spanish]. Universidad de Santiago de Compostela, Spain.

Díaz Pazos JA, Otero JC, 1993. [Contribución al conocimiento de los Hydraenidae de Galicia (NO Península Ibérica) (Insecta, Coleoptera) I. Estudio faunístico].[Article in Spanish]. Bol. R. Soc. Esp. Hist. Nat. Secc. Biol. 90:89-100.

Eyre MD, Carr R, MacBlane RP, Foster GN, 1992. The effects on the distribution of water beetle assemblages, adults and larvae (Coleoptera: Haliplidae, Dytiscidae, Hydrophilidae). Arch. Hydrobiol. 124:281-291.

Fairchild GW, Cruz J, Faulds AM, 2003. Microhabitat and landscape influences on aquatic beetle assemblages in a cluster of temporary and permanent ponds. J. N. Am. Benthol. Soc. 22:224-240.

Fery H, Fresneda J, 2007. [Los Hydradephaga (Coleoptera: Dytiscidae, Gyrinidae, Haliplidae, Noteridae, Paelobiidae) de la Península Ibérica e Islas Baleares de las colecciones de J. Fresneda y H. Fery].[Article in Spanish]. Boletin SEA 41:119-171.

Fery H, Nilsson AN, 1993. A revision of the Agabus chalconotus- and erichsoni- groups (Coleoptera, Dytiscidae) with a proposed phylogeny. Entomol. Scand. 24:79-108.

Foster GN, 2002. Beetling Bastions - The club tour of Central Spain 2002. Latissimus 15:13-14.

Foster GN, Foster AP, Eyre MD, Bilton DT, 1990. Classification of water beetle assemblages in arable fenland and ranking of sites in relation to conservation value. Freshwater Biol. 22:343-354.

Fresneda J, Hernando C, Rico E, 1990. [Datos para el conocimiento de los Hydradephaga (Coleoptera: Haliplidae, Dytiscidae) de la Península Ibérica].[Article in Spanish]. Llerda Ciencie 48:91-103.

García-Criado F, 1999. [Impacto de la minería del carbón sobre Hydraenidae y Elmidae (Coleoptera) en la cuenca del río Sil (León, España)]. [PhD Thesis in Spanish]. Universidad de León, Spain.

García-Criado F, Fernández-Aláez C, Fernández-Aláez M, 1999. Environmental variables influencing the distribution of Hydraenidae and Elmidae assemblages (Coleoptera) in a moderately-polluted river basin in north-western Spain. Eur. J. Entomol. 96:37-44.

Garrido J, Fernández M, Régil JA, 1994. Geographical distribution of Adephaga and Polyphaga (Coleoptera) in the Cantabrian Mountains (Spain): specific richness and analysis of the altitude factor. Arch. Hydrobiol. 131:353-380.

Garrido J, Munilla I, 2008. Aquatic Coleoptera and Hemiptera assemblages in three coastal lagoons of the NW Iberian Peninsula: assessment of conservation value and response to environmental factors. Aquat. Conserv. 18:557-569.

Garrido J, Sáinz-Cantero CE, 2004. [Diversidad de coleópteros 
acuáticos en la Península del Barbanza (Galicia, NW España) (Coleoptera, Adephaga y Polyphaga)].[Article in Spanish]. Nouv. Revue Ent. 21:49-64.

González J, 1992. [Los Coleópteros acuáticos de Galicia].[PhD Thesis in Spanish]. Universidad de Santiago de Compostela, Spain.

González J, Novoa F, 1995. [Coleópteros acuáticos de Galicia. II. Haliplidae, Noteridae, Gyrinidae, Hygrobiidae y Dytiscidae].[Article in Spanish]. Boln Asoc. Esp. Ent. 19:9-21.

Guareschi S, Gutiérrez-Cánovas C, Picazo F, Sánchez-Fernández D, Abellán P, Velasco J, Millán A, 2012. Aquatic macroinvertebrate biodiversity: patterns and surrogates in mountainous Spanish national parks. Aquat. Conserv. 22:598-615.

Hinden H, Oertli B, Menetrey N, Sager L, Lachavanne J-B, 2005. Alpine pond biodiversity: what are the related environmental variables? Aquat. Conserv. 15:613-624.

Jäch MA, Balke M, 2008. Global diversity of water beetles (Coleoptera) in freshwater. Hydrobiologia 595:419-442.

Kottek M, Grieser J, Beck C, Rudolf B, Rubel F, 2006. World Map of the Köppen-Geiger climate classification updated. Meteorol. Z. 15:259-263.

Martinoy M, Boix D, Sala J, Gascón S, Gifre J, Argerisch A, de la Barrera R, Brucet S, Badosa A, López-Flores R, Méndez M, Utgé JM, Quintana XD, 2006. Crustacean and aquatic insect assemblages in the Mediterranean coastal ecosystems of Empordà wetlands (NE Iberian Peninsula). Limnetica 25:665-682.

Mendoza G de, Catalán J, 2010. Lake macroinvertebrates and the altitudinal environmental gradient in the Pyrenees. Hydrobiologia 648:51-72.

Ministry of Agriculture, Alimentation and Environment (MAGRAMA), 2013. Accessed on: 08 February 2013. Available from: http://www.magrama.gob.es/

Mitsch WJ, Gosselink JG, 2000. Wetlands. J. Wiley \& Sons, New York: 920 pp.

Oertli B, Indermuehle N, Angélibert S, Hinden H, Stoll A, 2008. Macroinvertebrate assemblages in 25 high alpine ponds of the Swiss National Park (Cirque of Macun) and relation to environmental variables. Hydrobiologia 597:29-41.

Pérez-Bilbao A, Benetti CJ, Garrido J, 2010. [Coleópteros acuáticos (Adephaga: Gyrinidae, Haliplidae, Noteridae, Paelobiidae, Dytiscidae) en aguas estancadas protegidas de Galicia (NO España)]. [Article in Spanish]. Boln. Asoc. Esp. Ent. 34:267-289.

Pérez-Bilbao A, Benetti CJ, Garrido J, 2011. [Nuevas aportaciones al conocimiento de la familia Helophoridae en Galicia (NO España) (Coleoptera)]. [Article in Spanish]. Bull. Soc. Entomol. Fr. 116:35-41.

Pérez-Bilbao A, Garrido J, 2009. [Evaluación del estado de conservación de una zona LIC (Gándaras de Budiño, Red Natura 2000) usando los coleópteros acuáticos como indicadores].[Article in Spanish]. Limnetica 28:11-22.

Ribera I, Vogler AP, 2000. Habitat type as a determinant of species range sizes: the example of lotic-lentic differences in aquatic Coleoptera. Biol. J. Linn. Soc. 71:33-52.
Ribera I, Hernando C, Aguilera P, 1999. An annotated checklist of the Iberian water beetles (Coleoptera). Zapateri 8:43-111.

Sánchez-Fernández D, Abellán P, Mellado A, Velasco J, Millán A, 2006. Are water beetles good indicators of biodiversity in Mediterranean aquatic ecosystems? The case of the Segura River basin (SE Spain). Biodivers. Conserv. 15:4507-4520.

Sánchez-Fernández D, Abellán P, Velasco J, Millán A, 2004. Selecting areas to protect the biodiversity of aquatic ecosystems in a semiarid Mediterranean region using water beetles. Aquat. Conserv. 14:465-479.

Shine C, Klemm C, 1999. Wetlands, water and the law: using law to advance wetland conservation and wise use. IUCN, Gland: $332 \mathrm{pp}$.

Stenert C, Maltchik L, 2007. Influence of area, altitude and hydroperiod on macroinvertebrate communities in southern Brazil wetlands. Mar. Freshwater Res. 58:993-1001.

Studinski JM, Grubbs SA, 2007. Environmental factors affecting the distribution of aquatic invertebrates in temporary ponds in Mammoth Cave National Park, Kentucky, USA. Hydrobiologia 575:211-220.

Tews J, Brose U, Grimm V, Tielbörger K, Wichmann WC, Schwager M, Jeltsch F, 2004. Animal species diversity driven by habitat heterogeneity/diversity: the importance of keystone structures. J. Biogeogr. 31:79-92.

Trigal C, 2006. [Evaluación del estado ecológico de las lagunas esteparias de la depresión del Duero: ¿son los macroinvertebrados buenos indicadores?].[PhD Thesis in Spanish]. Universidad de León, Spain.

Valladares LF, Fernández-Aláez MC, Fernández-Aláez M, 1990. Influence of altitude in the distribution of the aquatic Hydrophiloidea (Coleoptera) in the Province of Leon (NW Spain). Limnetica 6:79-86.

Valladares LF, Garrido J, 2001. [Coleópteros acuáticos de los humedales asociados al Canal de Castilla (Palencia, España): Aspectos faunísticos y fenológicos (Coleoptera, Adephaga y Polyphaga)].[Article in Spanish]. Nouv. Revue Ent. 18:61-76.

Valladares LF, Garrido J, García-Criado F, 2002. The assemblages of aquatic Coleoptera from shallow lakes in the northern Iberian Meseta: influence of environmental variables. Eur. J. Entomol. 99:289-298.

Valladares LF, Ribera I, 1999. [Lista faunística y bibliográfica de los Hydrophiloidea acuáticos (Coleoptera) de la Península Ibérica e Islas Baleares. Listas de la flora y fauna de las aguas continentales de la Península Ibérica].[Book in Spanish]. Asociación Española de Limnología, Madrid: 114 pp.

Vivas S, Casas J, Pardo I, Robles S, Bonada N, Mellado N, Prat N, Alba-Tercedor J, Álvarez M, Bayo MM, Jáimez-Cuellar P, Suárez ML, Toro M, Vidal-Abarca MR, Zamora-Muñoz C, Moyá G, 2002. [Aproximación multivariante en la exploración de la tolerancia ambiental de las familias de macroinvertebrados de los ríos mediterráneos del proyecto GUADALMED].[Article in Spanish]. Limnetica 21:149-173.

Williams DD, 2006. The biology of temporary waters. Oxford University Press, Oxford: 348 pp. 